\title{
Counting surface branched covers
}

\author{
Carlo Petronio* $\quad$ Filippo Sarti
}

January 25, 2019

\begin{abstract}
To a branched cover $f$ between orientable surfaces one can associate a certain branch datum $\mathcal{D}(f)$, that encodes the combinatorics of the cover. This $\mathcal{D}(f)$ satisfies a compatibility condition called the RiemannHurwitz relation. The old but still partly unsolved Hurwitz problem asks whether for a given abstract compatible branch datum $\mathcal{D}$ there exists a branched cover $f$ such that $\mathcal{D}(f)=\mathcal{D}$. One can actually refine this problem and ask how many these f's exist, but one must of course decide what restrictions one puts on such $f$ 's, and choose an equivalence relation up to which one regards them. And it turns out that quite a few natural choices are possible. In this short note we carefully analyze all these choices and show that the number of actually distinct ones is only three. To see that these three choices are indeed different we employ Grothendieck's dessins d'enfant.
\end{abstract}

MSC (2010): 57M12.

Quite some energy has been devoted in ancient and more recent times to the computation of the so-called Hurwitz numbers, namely the numbers of equivalence classes of branched covers matching given branch data, see for instance [10, 11, 4, 7, 8, 9, 12, 17, 18. However there are several natural $a$ priori different ways to define the Hurwitz numbers exactly. In fact, one can decide to insist or not that a branched cover should respect the orientation, and to require or not that the branching points should be some fixed marked ones. Analogously, one can decide to include or not the orientation and/or the marking in the notion of equivalence. In this paper we carefully list and analyze all these possibilities, and we show that actually only three of

*Partially supported by INdAM through GNSAGA, by MIUR through PRIN "Real and Complex Manifolds: Geometry, Topology and Harmonic Analysis" and by UniPI through PRA 2018/22 "Geometria e Topologia delle varietà" 
them are really distinct. Our results and techniques have a rather elementary nature, but to the best of our knowledge no explicit account exists in the literature of all the possible ways under which the problem of counting surface branched covers could be faced. Two of the three different notions of equivalence however appear in [6], see the end of Section 3 for a discussion on this point.

\section{Branched covers, branch data, and the Hurwitz problem}

A surface branched cover is a map

$$
f: \widetilde{\Sigma} \rightarrow \Sigma
$$

where $\widetilde{\Sigma}$ and $\Sigma$ are closed and connected surfaces and $f$ is locally modeled on maps of the form

$$
(\mathbb{C}, 0) \ni z \mapsto z^{m} \in(\mathbb{C}, 0) .
$$

If $m>1$ the point 0 in the target $\mathbb{C}$ is called a branching point, and $m$ is called the local degree at the point 0 in the source $\mathbb{C}$. There are finitely many branching points, removing which, together with their preimages, one gets a genuine cover of some degree $d$. If there are $n$ branching points and we order them in some arbitrary fashion, the local degrees at the preimages of the $j$-th point form a partition $\pi_{j}$ of $d$, and we define

$$
\mathcal{D}(f)=\left(\widetilde{\Sigma}, \Sigma, d, n, \pi_{1}, \ldots, \pi_{n}\right)
$$

to be $a$ branch datum associated to $f$ (the datum is not unique because an order of the branching points has been chosen). If $\pi_{j}$ has length $\ell_{j}$, the following Riemann-Hurwitz relation holds:

$$
\chi(\widetilde{\Sigma})-\left(\ell_{1}+\ldots+\ell_{n}\right)=d(\chi(\Sigma)-n) .
$$

Let us now call abstract branch datum a 5-tuple

$$
\mathcal{D}=\left(\widetilde{\Sigma}, \Sigma, d, n, \pi_{1}, \ldots, \pi_{n}\right),
$$

a priori not coming from any $f$, and let us say it is compatible if it satisfies the Riemann-Hurwitz relation. (For a non-orientable $\widetilde{\Sigma}$ and/or $\Sigma$ this relation should actually be complemented with certain other necessary conditions, but we restrict to an orientable $\Sigma$ in this paper, so we do not spell out these conditions here.) 
The Hurwitz problem The very old Hurwitz problem asks which compatible abstract branch data $\mathcal{D}$ are realizable, namely, such that there exists $f: \widetilde{\Sigma} \rightarrow \Sigma$ with $\mathcal{D}(f)=\mathcal{D}$ for some ordering of the branching points of $f$, and which are exceptional (non-realizable). Several partial solutions to this problem have been obtained over the time, and we quickly mention here the fundamental [3], the survey [16], and the more recent [13, 14, 15, 2, 19]. In particular, for an orientable $\Sigma$ the problem has been shown to have a positive solution whenever $\Sigma$ has positive genus. When $\Sigma$ is the sphere $S$, many realizability and exceptionality results have been obtained (some of experimental nature), but the general pattern of what data are realizable remains elusive. One guiding conjecture in this context is that a compatible branch datum is always realizable if its degree is a prime number. It was actually shown in [3] that proving this conjecture in the special case of 3 branching points would imply the general case. This is why many efforts have been devoted in recent years to investigating the realizability of compatible branch data with base surface $\Sigma$ the sphere $S$ and having $n=3$ branching points. See in particular [14, 15] for some evidence supporting the conjecture.

\section{As many as 12 counting methods}

In this section and in the next one,

$$
\mathcal{D}(f)=\left(\widetilde{\Sigma}, \Sigma, d, n, \pi_{1}, \ldots, \pi_{n}\right)
$$

will be a fixed but arbitrary abstract compatible branch datum. Note that $\widetilde{\Sigma}$ and $\Sigma$ are supposed to be specific surfaces, they are not viewed up to any type of equivalence. Moreover we assume they have a fixed orientation, and we also fix $n$ points $p_{1}, \ldots, p_{n}$ in $\Sigma$. In the sequel we will call positive a map that respects the orientation.

We will say that a branched cover $f: \widetilde{\Sigma} \rightarrow \Sigma$ is:

- A realization of $\mathcal{D}$ if $\mathcal{D}(f)=\mathcal{D}$ for some order of the branching points;

- A positive realization of $\mathcal{D}$ if it is a realization and away from the branching points $f$ is a positive local homeomorphism;

- A marked realization if the branching points of $f$ are $p_{1}, \ldots, p_{n}$ and the partition of $d$ given by the local degrees of $f$ at the preimages of $p_{j}$ is $\pi_{j}$; 
- A marked positive realization if it is both a marked realization and a positive one.

Note that a marked realization of $\mathcal{D}$ is a realization. We denote by

$$
R(\mathcal{D}) \quad R_{+}(\mathcal{D}) \quad R_{*}(\mathcal{D}) \quad R_{*,+}(\mathcal{D})
$$

respectively the set of all the realizations of $\mathcal{D}$, the positive realizations, the marked realizations, the marked positive realizations. For all $m, s$ and $\mu, \sigma$ where $m, \mu$ are empty or $*$, except that $\mu$ must be empty if $m$ is, and $s, \sigma$ are empty or + , we define a quotient $\mathcal{R}_{m, s}^{\mu, \sigma}(\mathcal{D})$ of $R_{m, s}(\mathcal{D})$, where two realizations $f, f^{\prime}: \widetilde{\Sigma} \rightarrow \Sigma$ in $R_{m, s}$ are identified if there exists a commutative diagram

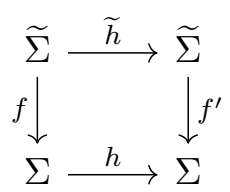

with $h, \widetilde{h}$ homeomorphisms, and:

- $h$ is the identity if $\mu=*$;

- $h$ and $\widetilde{h}$ are positive if $\sigma=+$.

So we have the quotients

$$
\begin{array}{llll}
\mathcal{R}(\mathcal{D}) & \mathcal{R}^{+}(\mathcal{D}) & \mathcal{R}_{+}(\mathcal{D}) & \mathcal{R}_{+}^{+}(\mathcal{D}) \\
\mathcal{R}_{*}(\mathcal{D}) & \mathcal{R}_{*}^{+}(\mathcal{D}) & \mathcal{R}_{*}^{*}(\mathcal{D}) & \mathcal{R}_{*}^{*++}(\mathcal{D}) \\
\mathcal{R}_{*,+}(\mathcal{D}) & \mathcal{R}_{*,+}^{+}(\mathcal{D}) & \mathcal{R}_{*,+}^{*}(\mathcal{D}) & \mathcal{R}_{*,+}^{*,+}(\mathcal{D})
\end{array}
$$

From the definitions it immediately follows that the following maps are defined:

- the quotient maps $q_{m, s}^{\mu}: \mathcal{R}_{m, s}^{\mu,+}(\mathcal{D}) \rightarrow \mathcal{R}_{m, s}^{\mu}(\mathcal{D})$;

- the quotient maps $c_{s}^{\sigma}: \mathcal{R}_{*, s}^{*, \sigma}(\mathcal{D}) \rightarrow \mathcal{R}_{*, s}^{\sigma}(\mathcal{D})$;

- the inclusions $j_{m}^{\mu, \sigma}: \mathcal{R}_{m,+}^{\mu, \sigma}(\mathcal{D}) \hookrightarrow \mathcal{R}_{m}^{\mu, \sigma}(\mathcal{D})$;

- the forgetful maps $o_{s}^{\sigma}: \mathcal{R}_{*, s}^{\sigma}(\mathcal{D}) \rightarrow \mathcal{R}_{s}^{\sigma}(\mathcal{D})$.

Therefore we get the following commutative diagram 


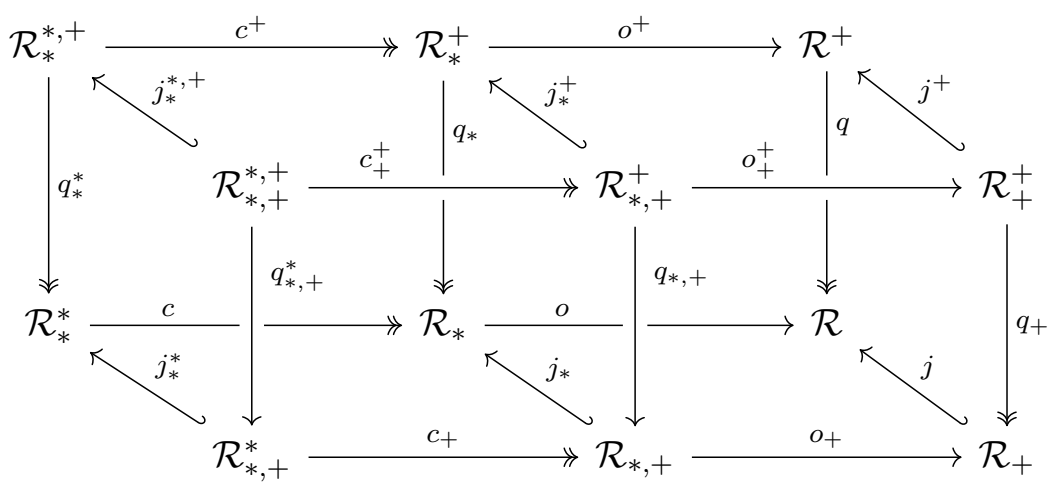

where to save space we have omitted any explicit reference to $\mathcal{D}$.

\section{At most three methods are distinct}

In this section we show that several of the maps in the above commutative diagram are actually bijections or otherwise easily understood.

Proposition 3.1. $q_{*,+}^{*}$ is a bijection.

Proof. If $f, f^{\prime} \in R_{*,+}$ are equivalent in $\mathcal{R}_{*,+}^{*}(\mathcal{D})$ then there exists a commutative diagram as 11 with $h$ the identity. But $f$ and $f^{\prime}$ are positive, so $\widetilde{h}$ also is, whence $f$ and $f^{\prime}$ are equivalent in $\mathcal{R}_{*,+}^{*,+}(\mathcal{D})$.

Proposition 3.2. $j, j_{*}$ and $j_{*}^{*}$ are bijections.

Proof. We spell out the argument for $j$, the other two cases are identical. Fix a negative involutive automorphism $\rho$ of $\widetilde{\Sigma}$. Our aim is to define an inverse $\varphi: \mathcal{R}(\mathcal{D}) \rightarrow \mathcal{R}_{+}(\mathcal{D})$ of $j$. To do so for $f \in R(\mathcal{D})$ we set

$$
\varphi([f])= \begin{cases}{[f]} & \text { if } f \in R_{+}(\mathcal{D}) \\ {[f \circ \rho]} & \text { if } f \notin R_{+}(\mathcal{D})\end{cases}
$$

and we prove the following:

FACT: $\varphi$ IS WELL-DEFINED Note first that $f \circ \rho \in R_{+}(\mathcal{D})$ if $f \notin R_{+}(\mathcal{D})$, so it makes sense to take $[f \circ \rho] \in \mathcal{R}_{+}(\mathcal{D})$. Assume now $f, f^{\prime} \in R(\mathcal{D})$ are equivalent in $\mathcal{R}(\mathcal{D})$, so there exists a commutative diagram as (1). We have four cases: 
- If $f, f^{\prime} \in R_{+}(\mathcal{D})$ the same diagram shows that $f$ and $f^{\prime}$ are equivalent in $\mathcal{R}_{+}(\mathcal{D})$;

- If $f \in R_{+}(\mathcal{D})$ and $f^{\prime} \notin R_{+}(\mathcal{D})$ then $f$ and $f^{\prime} \circ \rho$ are equivalent in $\mathcal{R}_{+}(\mathcal{D})$ because we have the commutative diagram

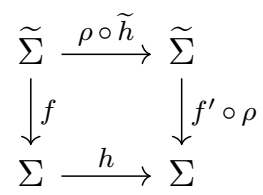

(recall that $\rho$ is involutive, so $\rho^{2}$ is the identity of $\widetilde{\Sigma}$ );

- If $f \notin R_{+}(\mathcal{D})$ and $f^{\prime} \in R_{+}(\mathcal{D})$ then $f \circ \rho$ and $f^{\prime}$ are equivalent in $\mathcal{R}_{+}(\mathcal{D})$ because we have the commutative diagram

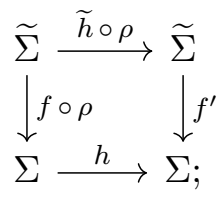

- If $f, f^{\prime} \notin R_{+}(\mathcal{D})$ then $f \circ \rho$ and $f^{\prime} \circ \rho$ are equivalent in $\mathcal{R}_{+}(\mathcal{D})$ because we have the commutative diagram

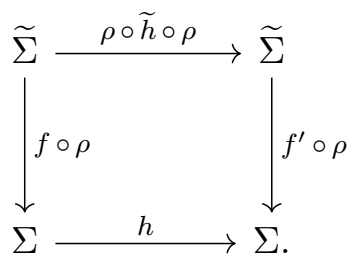

FACT: $\varphi$ IS A LEFT INVERSE OF $j$ This is immediate: for $f \in R_{+}(\mathcal{D})$ we have $j([f])=[f] \in \mathcal{R}(\mathcal{D})$, whence $(\varphi \circ j)([f])=[f] \in \mathcal{R}_{+}(\mathcal{D})$.

FACT: $\varphi$ IS A RIGHT INVERSE OF $j$ Given $f \in R(\mathcal{D})$ we have two cases; if $f \in R_{+}(\mathcal{D})$ then $\varphi([f])=[f] \in \mathcal{R}_{+}(\mathcal{D})$, so $(j \circ \varphi)([f])=[f] \in \mathcal{R}(\mathcal{D})$; if $f \notin R_{+}(\mathcal{D})$ then $\varphi([f])=[f \circ \rho] \in \mathcal{R}_{+}(\mathcal{D})$, so $(j \circ \varphi)([f])=[f \circ \rho] \in \mathcal{R}(\mathcal{D})$, but we actually have that $f$ and $f \circ \rho$ are equivalent in $\mathcal{R}(\mathcal{D})$, because we have the commutative diagram 


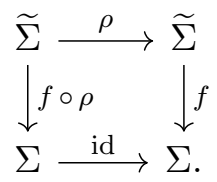

The proof is complete.

Proposition 3.3. $o, o_{+}, o^{+}$and $o_{+}^{+}$are bijections.

Proof. We begin with $o$ and we construct its inverse $\varphi$. Given $f \in R(\mathcal{D})$, suppose that $f$ has branching points $x_{1}, \ldots, x_{n}$ ordered so that the local degrees of $f$ over $x_{j}$ form the partition $\pi_{j}$ of $d$. We then choose an automorphism $g$ of $\Sigma$ such that $g\left(x_{j}\right)=p_{j}$ for all $j$ and set $\varphi\left([f]=[g \circ f] \in \mathcal{R}_{*}(\mathcal{D})\right.$. We have the following:

FACT: $\varphi$ IS WELL-DEFINED Note first that indeed $g \circ f \in R_{*}(\mathcal{D})$. Moreover $[g \circ f] \in \mathcal{R}_{*}(\mathcal{D})$ is independent of $g$, because if $g^{\prime}$ is another automorphism of $\Sigma$ such that $g^{\prime}\left(x_{j}\right)=p_{j}$ for all $j$ we have the commutative diagram

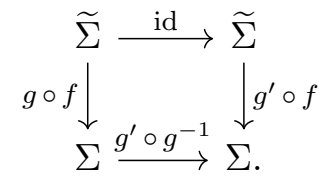

Suppose now that $f, f^{\prime} \in R(\mathcal{D})$ are equivalent in $\mathcal{R}(\mathcal{D})$, so there exists a commutative diagram as $(1)$; let $x_{1}, \ldots, x_{n}$ and $x_{1}^{\prime}, \ldots, x_{n}^{\prime}$ be the branching points of $f$ and $f^{\prime}$ ordered so that the local degrees of $f$ over $x_{j}$ and those of $f^{\prime}$ over $x_{j}^{\prime}$ form the partition $\pi_{j}$ of $d$. Take automorphisms $g$ and $g^{\prime}$ of $\Sigma$ such that $g\left(x_{j}\right)=g^{\prime}\left(x_{j}^{\prime}\right)=p_{j}$ for all $j$. Then $g \circ f$ and $g^{\prime} \circ f^{\prime}$ are equivalent in $\mathcal{R}_{*}(\mathcal{D})$ because we have the commutative diagram

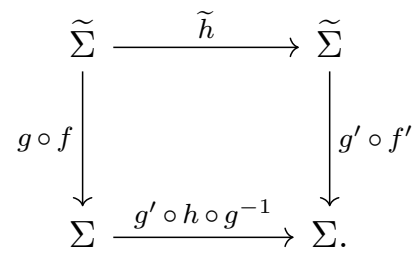

FACT: $\varphi$ IS A LEFT InVERSE OF $o$ Given $f \in R_{*}(\mathcal{D})$ we know that the branching points of $f$ are $p_{1}, \ldots, p_{n}$ with associated partitions $\pi_{1}, \ldots, \pi_{n}$, so in the definition of $\varphi$ we can take $g=\mathrm{id}$ and it readily follows that

$$
(\varphi \circ o)([f])=\varphi(o([f]))=\varphi([f])=[\operatorname{id} \circ f]=[f] .
$$


FACT: $\varphi$ IS A RIGHT InVERSE OF $o$ Take $f \in R(\mathcal{D})$ and suppose that $\varphi([f])$ is defined as $[g \circ f]$. Then

$$
(o \circ \varphi)[f]=o(\varphi([f]))=o([g \circ f])=[g \circ f]
$$

but $g \circ f$ is equivalent to $f$ in $\mathcal{R}(\mathcal{D})$ because we have the commutative diagram

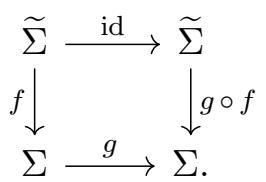

This concludes the argument for $o$. Repeating it for $o_{+}$only requires to remark that the automorphism $g$ of $\Sigma$ used to define $\varphi(f)$ can always be chosen to be positive. The extension to $o^{+}$and $o_{+}^{+}$is straight-forward.

Proposition 3.4. There exist natural $2: 1$ projections

$$
\mathcal{R}^{+}(\mathcal{D}) \rightarrow \mathcal{R}_{+}^{+}(\mathcal{D}) \quad \mathcal{R}_{*}^{*,+}(\mathcal{D}) \rightarrow \mathcal{R}_{*,+}^{*,+}(\mathcal{D})
$$

having $j^{+}$and $j_{+}^{*,+}$ as sections, so $\mathcal{R}^{+}(\mathcal{D})$ can be identified to two copies of $\mathcal{R}_{+}^{+}(\mathcal{D})$, and $\mathcal{R}_{*}^{*,+}(\mathcal{D})$ can be identified to two copies of $\mathcal{R}_{*,+}^{*,+}(\mathcal{D})$.

Proof. As in the proof of Proposition 3.2 we fix a negative involutive automorphism $\rho$ of $\widetilde{\Sigma}$. We spell out the proof for $j^{+}$; the version for $j_{+}^{*++}$ is identical. For $f \in R(\mathcal{D})$ we set

$$
\varphi([f])= \begin{cases}{[f]} & \text { if } f \in R_{+}(\mathcal{D}) \\ {[f \circ \rho]} & \text { if } f \notin R_{+}(\mathcal{D})\end{cases}
$$

and we have the following:

FACT: $\varphi: \mathcal{R}^{+}(\mathcal{D}) \rightarrow \mathcal{R}_{+}^{+}(\mathcal{D})$ IS WELL-DEFINED For $f \notin R_{+}(\mathcal{D})$ we have $f \circ \rho \in R_{+}(\mathcal{D})$, so it makes sense to take $[f \circ \rho] \in \mathcal{R}_{+}^{+}(\mathcal{D})$. If $f, f^{\prime} \in R(\mathcal{D})$ are equivalent in $\mathcal{R}^{+}(\mathcal{D})$ then there exists a commutative diagram as (1) with $h$ and $\widetilde{h}$ positive. It follows that either $f, f^{\prime} \in R_{+}(\mathcal{D})$ or $f, f^{\prime} \notin R_{+}(\mathcal{D})$. In the former case the same diagram shows that $f$ and $f^{\prime}$ are equivalent in $\mathcal{R}_{+}^{+}(\mathcal{D})$. In the latter case a commutative diagram as (2) shows that $f \circ \rho$ and $f^{\prime} \circ \rho$ are equivalent in $\mathcal{R}_{+}^{+}(\mathcal{D})$.

FACT: $\varphi$ IS $2: 1 \quad$ For $g \in R_{+}(\mathcal{D})$ we have that $\varphi^{-1}([g])$ certainly contains $[g]$ and $[g \circ \rho]$. Conversely, suppose that $\varphi([f])=[g]$. We have two cases. Either $f \in R_{+}(\mathcal{D})$, whence $\varphi([f])=[f]$, which implies that $[f]=[g]$ in $\mathcal{R}_{+}^{+}(\mathcal{D})$ and hence in particular in $\mathcal{R}^{+}(\mathcal{D})$. Or $f \notin R_{+}(\mathcal{D})$, so $\varphi([f])=[f \circ \rho]$, whence $[f \circ \rho]=[g]$ in $\mathcal{R}_{+}^{+}(\mathcal{D})$; this implies that we have a commutative diagram 


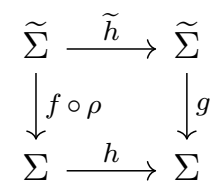

whence

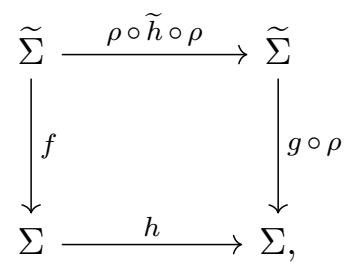

therefore $[f]=[g \circ \rho]$ in $\mathcal{R}^{+}(\mathcal{D})$. Now we note that $g$ and $g \circ \rho$ cannot be equivalent in $\mathcal{R}^{+}(\mathcal{D})$ and the claimed fact is proved.

FACT: $j^{+}$IS A SECTION OF $\varphi$ This is immediate, because for $g \in R_{+}(\mathcal{D})$ we have $j^{+}([g])=[g]$ and $\varphi([g])=[g]$.

The results proved so far imply that of the 12 potentially distinct $\mathcal{R}_{m, s}^{\mu, \sigma}(\mathcal{D})$ only three remain to understand, because

$$
\begin{aligned}
& \mathcal{R}(\mathcal{D})=\mathcal{R}_{+}(\mathcal{D}) \quad=\mathcal{R}_{*,+}(\mathcal{D}) \quad=\mathcal{R}_{*}(\mathcal{D}) \\
& \mathcal{R}_{+}^{+}(\mathcal{D})=\mathcal{R}_{*,+}^{+}(\mathcal{D}) \\
& \mathcal{R}_{*,+}^{*,+}(\mathcal{D})=\mathcal{R}_{*,+}^{*}(\mathcal{D})=\mathcal{R}_{*}^{*}(\mathcal{D}) \\
& \mathcal{R}_{*}^{+}(\mathcal{D})=\mathcal{R}^{+}(\mathcal{D})=2 \times \mathcal{R}_{+}^{+}(\mathcal{D}) \\
& \mathcal{R}_{*}^{*,+}(\mathcal{D})=2 \times \mathcal{R}_{*,+}^{*,+}(\mathcal{D}) \text {. }
\end{aligned}
$$

Note that each $\mathcal{R}_{m, s}^{\mu, \sigma}(\mathcal{D})$ is a finite set (see also Section 4) and does not carry any significant algebraic structure, so only its cardinality actually matters. In the rest of the paper we will concentrate on

$$
\mathcal{R}_{*,+}^{*,+}(\mathcal{D}) \quad \mathcal{R}_{+}^{+}(\mathcal{D}) \quad \mathcal{R}(\mathcal{D})
$$

and prove that they can indeed differ from each other. Note that by construction

$$
\#\left(\mathcal{R}_{*,+}^{*,+}(\mathcal{D})\right) \geqslant \#\left(\mathcal{R}_{+}^{+}(\mathcal{D})\right) \geqslant \#(\mathcal{R}(\mathcal{D}))
$$

and they can only vanish simultaneously.

We remark here that our $\mathcal{R}_{*,+}^{*,+}(\mathcal{D})$ is called in [6] the set of rigid equivalence classes of branched covers matching $\mathcal{D}$, while $\mathcal{R}_{+}^{+}(\mathcal{D})$ is called the set of flexible equivalence classes. From this viewpoint, we could call $\mathcal{R}(\mathcal{D})$ the set of very flexible equivalence classes. 


\section{Dessins d'enfant}

Graphs on surfaces have been used for a long time to understand surface branched covers, see [6]; their version for the case where $\Sigma$ is the sphere $S$ and the number $n$ of branching point is 3 has been popularized by Grothendieck under the name of dessins d'enfant, and pushed to its most ultimate algebraic consequences, see [5] and [1]. In this paper we will adopt a purely topological viewpoint, neglecting the algebraic one altogether.

Let us call graph a cellular 1-complex $\Gamma$, consisting of vertices and edges (we do not insist that $\Gamma$ should be simplicial, so loops and multiple edges are allowed). We say that $\Gamma$ is bipartite if its vertices are coloured black and white, and every edge joins black to white. We then call dessin d'enfant on $\widetilde{\Sigma}$ a bipartite graph $\Gamma$ embedded in $\widetilde{\Sigma}$ so that the complement of $\Gamma$ consists of open discs, called regions. The length of a region of $\Gamma$ is now the number of black (or white) vertices it is incident to (with multiplicity).

In the rest of this section we fix a branch datum $\mathcal{D}$ with base surface $\Sigma$ the sphere $S$ and $n=3$ branching points, so

$$
\mathcal{D}=\left(\widetilde{\Sigma}, S, d, 3, \pi_{1}, \pi_{2}, \pi_{3}\right) .
$$

The results we prove here could be extracted from the literature [6], but we spell them out for the sake of completeness. We will denote by $G(\mathcal{D})$ the

set of dessins d'enfant $\Gamma$ on $\widetilde{\Sigma}$ such that the valences of the black vertices of $\Gamma$ give the partition $\pi_{1}$ of $d$, the valences of the white vertices give $\pi_{2}$, while the lengths of the regions of $\Gamma$ give $\pi_{3}$. We define the quotient $\mathcal{G}_{*}(\mathcal{D})$ of $G(\mathcal{D})$ under the action of the positive automorphisms of $\widetilde{\Sigma}$.

Proposition 4.1. There is a natural bijection between $\mathcal{R}_{*,+}^{*,+}(\mathcal{D})$ and $\mathcal{G}_{*}(\mathcal{D})$.

Proof. Take $f \in R_{*,+}(\mathcal{D})$ and recall that the branching points of $f$ are the fixed points $p_{1}, p_{2}, p_{3}$, with associated partitions $\pi_{1}, \pi_{2}, \pi_{3}$ of $d$, in this order. Now choose in $S$ a simple arc $\alpha$ joining $p_{1}$ to $p_{2}$, paint $p_{1}$ black and $\pi_{2}$ white, and define $\Gamma=f^{-1}(\alpha)$, with vertex colours lifted through $f$. Since $\alpha$ is unique up to isotopy fixed on $p_{1}$ and $p_{2}$, the dessin d'enfant $\Gamma$ is well-defined up to positive automorphisms of $\widetilde{\Sigma}$. Moreover, by the definition of the equivalence relation giving $\mathcal{R}_{*,+}^{*,+}(\mathcal{D})$, we see that $[\Gamma] \in \mathcal{G}_{*}(\mathcal{D})$ only depends on $[f] \in \mathcal{R}_{*,+}^{*,+}(\mathcal{D})$.

Conversely, given $\Gamma \in G(\mathcal{D})$, we can choose in $S$ a simple arc $\alpha$ joining $p_{1}$ to $p_{2}$, and map $\Gamma$ continuously to $\alpha$ sending the black vertices to $p_{1}$, the white vertices to $p_{2}$, and each edge bijectively onto $\alpha$. We can now extend 
this map continuously to each complementary region $\Omega$ of $\Gamma$, in such a way that $\Omega$ is mapped onto $S$ and there is only one branching point in the interior of $\Omega$, mapped to $p_{3}$ with local degree equal to the length of $\Omega$. Patching these extensions together we get a branched cover $f$ in $R_{*,+}(\mathcal{D})$, and it is a routine matter to check that $[f] \in \mathcal{R}_{*,+}^{*,+}(\mathcal{D})$ only depends on $[\Gamma] \in \mathcal{G}_{*}(\mathcal{D})$.

The two maps $[f] \mapsto[\Gamma]$ and $[\Gamma] \mapsto[f]$ are the inverse of each other, whence the conclusion.

We next define a further quotient $\mathcal{G}_{+}(\mathcal{D})$ where two elements of $\mathcal{G}_{*}(\mathcal{D})$ are identified if two of their representatives can be obtained from each other by a combination of the following moves $\Gamma \mapsto \Gamma^{\prime}$ :

- $\Gamma^{\prime}$ equals $\Gamma$ as a graph, but the black and white colours of the vertices are switched;

- $\Gamma^{\prime}$ has the same black vertices as $\Gamma$, one white vertex in the interior of each complementary region $\Omega$ of $\Gamma$, and disjoint edges contained in $\Omega$ joining this white vertex to the black vertices adjacent to $\Omega$.

Note that a single move $\Gamma \mapsto \Gamma^{\prime}$ might lead from a dessin in $G(\mathcal{D})$ to one outside $G(\mathcal{D})$, but then a further move $\Gamma \mapsto \Gamma^{\prime}$ might lead back to $G(\mathcal{D})$. Note also that if $\pi_{1}, \pi_{2}, \pi_{3}$ are distinct then $\mathcal{G}_{+}(\mathcal{D})=\mathcal{G}_{*}(\mathcal{D})$.

Proposition 4.2. There is a natural bijection between $\mathcal{R}_{+}^{+}(\mathcal{D})$ and $\mathcal{G}_{+}(\mathcal{D})$.

Proof. Recall that we have a natural surjection $c_{+}^{+}: \mathcal{R}_{*,+}^{*,+}(\mathcal{D}) \rightarrow \mathcal{R}_{*,+}^{+}(\mathcal{D})$ and that $\mathcal{R}_{+}^{+}(\mathcal{D})$ is identified to $\mathcal{R}_{*,+}^{+}(\mathcal{D})$ via $o_{+}^{+}$. Take $f, f^{\prime} \in R_{*,+}(\mathcal{D})$ and suppose that $c_{+}^{+}([f])=c_{+}^{+}\left(\left[f^{\prime}\right]\right)$. Then there exists a diagram as $(1)$ with positive $h, \widetilde{h}$. Choose in $S$ arcs $\alpha$ and $\alpha^{\prime}$ joining $p_{1}$ to $p_{2}$, with a black end at $p_{1}$ and a white end at $p_{2}$, so the equivalence classes in $\mathcal{G}_{*}(\mathcal{D})$ of $\Gamma=f^{-1}(\alpha)$ and $\Gamma^{\prime}=f^{\prime-1}\left(\alpha^{\prime}\right)$ correspond to the elements $[f]$ and $\left[f^{\prime}\right]$ of $\mathcal{R}_{*,+}^{*,+}(\mathcal{D})$. Now $h^{-1}\left(\alpha^{\prime}\right)$ need not be isotopic to $\alpha$ with isotopy fixed at the ends, because it will have its black end at some $p_{i_{1}}$ and its white end at some $p_{i_{2}}$ (but note that we must have $\pi_{i_{1}}=\pi_{1}$ and $\pi_{i_{2}}=\pi_{2}$ ). However $\alpha^{\prime}$ becomes $\alpha$, up to isotopy fixed at the ends, by a combination of the following moves applied to an arc:

- Switch the colours of the ends of the arc;

- Supposing the black end is at $p_{1}$ and the white end is at $p_{2}$, replace the arc by one with black end at $p_{1}$ and white end at $p_{3}$. 
This implies that $\widetilde{h}^{-1}\left(\Gamma^{\prime}\right)$ becomes $\Gamma$ by a combination of the moves defining the equivalence in $\mathcal{G}_{+}(\mathcal{D})$, so $[\Gamma]=\left[\Gamma^{\prime}\right]$ in $\mathcal{G}_{+}(\mathcal{D})$. The same construction shows that if $[\Gamma]=\left[\Gamma^{\prime}\right]$ in $\mathcal{G}_{+}(\mathcal{D})$ then $c_{+}^{+}([f])=c_{+}^{+}\left(\left[f^{\prime}\right]\right)$, whence the conclusion.

As a final step, we take the quotient $\mathcal{G}(\mathcal{D})$ of $\mathcal{G}_{+}(\mathcal{D})$ by allowing the action of the negative automorphisms of $\widetilde{\Sigma}$ as well. Then we easily have:

Proposition 4.3. There is a natural bijection between $\mathcal{R}(\mathcal{D})$ and $\mathcal{G}(\mathcal{D})$.

\section{$5 \quad$ Precisely three methods are distinct}

By the results of the previous section, all we must show to conclude that

$$
\mathcal{R}_{*,+}^{*,+}(\mathcal{D}) \quad \mathcal{R}_{+}^{+}(\mathcal{D}) \quad \mathcal{R}(\mathcal{D})
$$

are distinct in some cases, is to show that each of the natural projections

$$
\mathcal{G}_{*}(\mathcal{D}) \rightarrow \mathcal{G}_{+}(\mathcal{D}) \quad \mathcal{G}_{+}(\mathcal{D}) \rightarrow \mathcal{G}(\mathcal{D})
$$

can fail to be a bijection. Note first that given

$$
\mathcal{D}=\left(\widetilde{\Sigma}, S, d, 3, \pi_{1}, \pi_{2}, \pi_{3}\right)
$$

there are finitely many abstract bipartite graphs with valences of the black vertices the entries of $\pi_{1}$ and valences of the white vertices the entries of $\pi_{2}$. Each of these graphs embeds in $\widetilde{\Sigma}$ in only a finite number of ways up to automorphisms of $\widetilde{\Sigma}$, so $\mathcal{G}_{*}(\mathcal{D})$ is always a finite set. Even if we will not need this here, we note that variants of the argument based on dessins d'enfant prove that $\mathcal{R}_{*,+}^{*,+}(\mathcal{D})$ is always a finite set, so $\mathcal{R}_{+}^{+}(\mathcal{D})$ and $\mathcal{R}(\mathcal{D})$ also are.

Let us now consider the branch datum

$$
\mathcal{D}=(S, S, 7,3,[3,2,1,1],[3,2,1,1],[7]) .
$$

An easy enumeration argument proves that $\mathcal{G}_{*}(\mathcal{D})$ has 9 elements, with representatives $\Gamma_{1}, \ldots, \Gamma_{9}$ shown in Fig. 1 .

Now one sees that the graphs $\Gamma_{2}$ and $\Gamma_{3}$ are obtained from each other by a switch of colours, and the same happens for $\Gamma_{6}$ and $\Gamma_{9}$, and for $\Gamma_{7}$ and $\Gamma_{8}$. Note that only the switch of colours is relevant to understand the projection $\mathcal{G}_{*}(\mathcal{D}) \rightarrow \mathcal{G}_{+}(\mathcal{D})$, because $\pi_{1}=\pi_{2} \neq \pi_{3}$, so we conclude that $\mathcal{G}_{+}(\mathcal{D})$ has 6 elements. We must now analyze what equivalence classes in $\mathcal{G}_{+}(\mathcal{D})$ are 
$\Gamma_{1}$
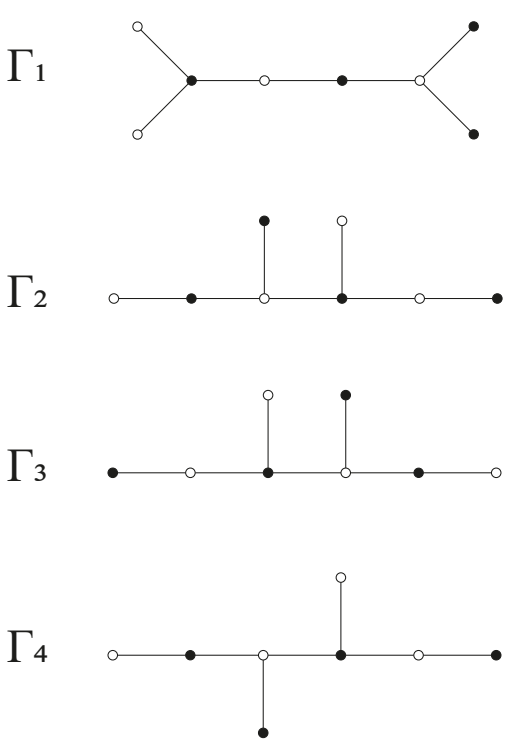
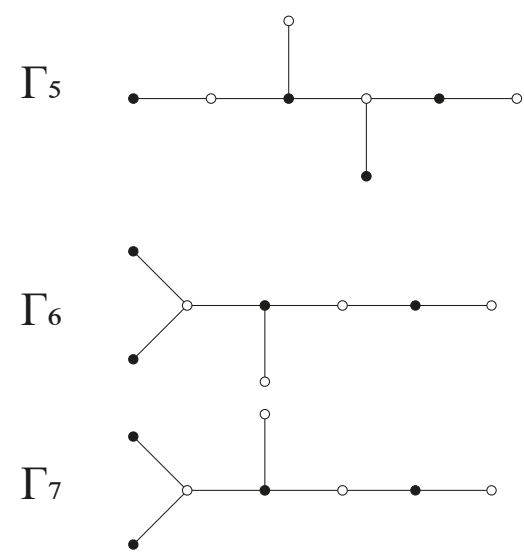

$\Gamma_{8}$

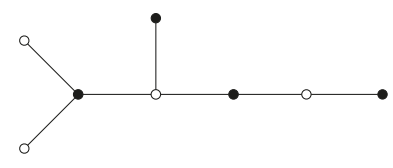

$\Gamma_{9}$

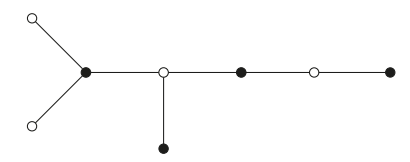

Figure 1: Representatives of $\mathcal{G}_{*}(S, S, 7,3,[3,2,1,1],[3,2,1,1]$, [7]).

related by a reflection in $S$, and we see that it happens precisely for those of $\Gamma_{4}$ and $\Gamma_{5}$, and for those of $\Gamma_{6}$ and $\Gamma_{7}$. This implies that $\mathcal{G}(\mathcal{D})$ has 4 elements. Therefore

$$
\begin{aligned}
& \#\left(\mathcal{R}_{*,+}^{*+}(S, S, 7,3,[3,2,1,1],[3,2,1,1],[7])\right)=9 \\
>\quad & \#\left(\mathcal{R}_{+}^{+}(S, S, 7,3,[3,2,1,1],[3,2,1,1],[7])\right)=6 \\
>\quad & \#(\mathcal{R}(S, S, 7,3,[3,2,1,1],[3,2,1,1],[7]))=4 .
\end{aligned}
$$

Without providing the details we also note that in the relation

$$
\#\left(\mathcal{R}_{*,+}^{*,+}(\mathcal{D})\right) \geqslant \#\left(\mathcal{R}_{+}^{+}(\mathcal{D})\right) \geqslant \#(\mathcal{R}(\mathcal{D}))
$$

all the possibilities for the $\geqslant$ relations occur in reality; for instance

$$
\begin{aligned}
& \#\left(\mathcal{R}_{*,+}^{*,+}(S, S, 7,3,[7],[4,1,1,1],[3,2,1,1])\right) \\
= & \#\left(\mathcal{R}_{+}^{+}(S, S, 7,3,[7],[4,1,1,1],[3,2,1,1])\right)=3 \\
>\quad & \#(\mathcal{R}(S, S, 7,3,[7],[4,1,1,1],[3,2,1,1]))=2,
\end{aligned}
$$




$$
\begin{aligned}
& \#\left(\mathcal{R}_{*,+}^{*+}(S, S, 7,3,[3,3,1],[3,3,1],[4,2,1])\right)=4 \\
>\quad & \#\left(\mathcal{R}_{+}^{+}(S, S, 7,3,[3,3,1],[3,3,1],[4,2,1])\right) \\
= & \#(\mathcal{R}(S, S, 7,3,[3,3,1],[3,3,1],[4,2,1]))=2, \\
& \#\left(\mathcal{R}_{*,+}^{*,+}(S, S, 8,3,[4,2,2],[2,2,1,1,1,1],[8])\right) \\
= & \#\left(\mathcal{R}_{+}^{+}(S, S, 8,3,[4,2,2],[2,2,1,1,1,1],[8])\right) \\
= & \#(\mathcal{R}(S, S, 8,3,[4,2,2],[2,2,1,1,1,1],[8]))=3 .
\end{aligned}
$$

To conclude we note that the Hurwitz numbers computed in [10, 11] are $\#\left(\mathcal{R}_{*,+}^{*,+}(\mathcal{D})\right)$, while those computed in [17, 18] are $\#(\mathcal{R}(\mathcal{D}))$.

\section{References}

[1] P. B. Cohen (now P. Tretkoff), Dessins denfant and Shimura varieties, In: "The Grothendieck Theory of Dessins dEnfants," (L. Schneps, ed.), London Math. Soc. Lecture Notes Series, Vol. 200, Cambridge University Press, 1994, pp. 237-243.

[2] P. Corvaja - C. Petronio - U. Zannier, On certain permutation groups and sums of two squares, Elem. Math. 67 (2012), 169-181.

[3] A. L. Edmonds - R. S. Kulkarni - R. E. Stong, Realizability of branched coverings of surfaces, Trans. Amer. Math. Soc. 282 (1984), 773-790.

[4] I. P. Goulden - J. H. KWAK - J. Lee, Distributions of regular branched surface coverings, European J. Combin. 25 (2004), 437-455.

[5] A. Grothendieck, Esquisse d'un programme (1984). In: "Geometric Galois Action" (L. Schneps, P. Lochak eds.), 1: "Around Grothendieck's Esquisse d'un Programme," London Math. Soc. Lecture Notes Series, Vol. 242, Cambridge Univ. Press, 1997, pp. 5-48.

[6] S. K. Lando - A. K. Zvonkin, "Graphs on Surfaces and their Applications," Encyclopaedia Math. Sci. Vol. 141, Springer, Berlin, 2004.

[7] J. H. Kwak, A. Mednykh, Enumeration of branched coverings of closed orientable surfaces whose branch orders coincide with multiplicity, Studia Sci. Math. Hungar. 44 (2007), 215-223. 
[8] J. H. KWAK, A. MEDNYKH, Enumerating branched coverings over surfaces with boundaries, European J. Combin. 25 (2004), 23-34.

[9] J. H. Kwak, A. Mednykh, V. Liskovets, Enumeration of branched coverings of nonorientable surfaces with cyclic branch points, SIAM J. Discrete Math. 19 (2005), 388-398.

[10] A. D. Mednykh, On the solution of the Hurwitz problem on the number of nonequivalent coverings over a compact Riemann surface (Russian), Dokl. Akad. Nauk SSSR 261 (1981), 537-542.

[11] A. D. Mednykh, Nonequivalent coverings of Riemann surfaces with a prescribed ramification type (Russian), Sibirsk. Mat. Zh. 25 (1984), 120-142.

[12] S. Monni, J. S. Song, Y. S. Song, The Hurwitz enumeration problem of branched covers and Hodge integrals, J. Geom. Phys. 50 (2004), 223256.

[13] F. Pakovich, Solution of the Hurwitz problem for Laurent polynomials, J. Knot Theory Ramifications 18 (2009), 271-302.

[14] M. A. Pascali - C. Petronio, Surface branched covers and geometric 2-orbifolds, Trans. Amer. Math. Soc. 361 (2009), 5885-5920

[15] M. A. Pascali - C. Petronio, Branched covers of the sphere and the prime-degree conjecture, Ann. Mat. Pura Appl. 191 (2012), 563-594.

[16] E. Pervova - C. Petronio, Realizability and exceptionality of candidate surface branched covers: methods and results, Seminari di Geometria 2005-2009, Università degli Studi di Bologna, Dipartimento di Matematica, Bologna 2010, pp. 105-120.

[17] C. Petronio, Explicit computation of some families of Hurwitz numbers, European J. Combin. 75 (2018), 136-151.

[18] C. Petronio, Explicit computation of some families of Hurwitz numbers, II, preprint arXiv:1807.11067.

[19] J. Song - B. Xu, On rational functions with more than three branch points, arXiv:1510.06291 